\title{
The Impact of Grave's Disease Hyperthyroidism on Grey Matter Atrophy
}

\author{
Julia E. Carneiro*, Mateus H. Nogueira, Tatila M. Lopes, Tamires A. Zanão, Marcos Tambascia, Fernando \\ Cendes, Denise E. Zantut-Wittmann, Clarissa L. Yasuda.
}

\begin{abstract}
Graves' disease is an autoimmune disease in which the production of thyroid hormones causes the state of thyrotoxicity. This state can cause tissue damage and may alter cognitive alterations and psychiatric and neuropsychological functions. However, it is not clear in the literature the relation of this type of deficit and thyrotoxicity, or if these changes are permanent or if they are caused by another pathology. Our goal in this study is quantitatively evaluate the gray matter atrophy of patients with euthyroidism and hyperthyroidism of autoimmune etiology (Graves' disease) using Voxel-based Morphometry.
\end{abstract}

\section{Key words: \\ Hyperthyroidism, Grave's disease, Voxel-based Morphometry.}

\section{Introduction}

Graves' disease is an autoimmune disease, in which auto-antibodies (TSI, thyroidstimulating immunoglobulin) stimulate TSH receptors, which in turn stimulate the production of thyroid hormones causing the state of thyrotoxicity. Thyroid hormone, when in the state of thyrotoxicity, can cause tissue damage and may alter psychiatric and neuropsychological functions, cognitive alterations such as memory deficits.

Studies show that receptors of thyroid hormone in the limbic system are affected by excess hormone by modifying an activity of neurotransmitters such as a serotonin and a dopamine, that contributes to neuropsychiatric deficits of thyrotoxicity. However, the endocrine mechanism of this process is still not clear, nor is it clear in the literature the relation of this type of deficit and thyrotoxicity, or if these changes are permanent or if they are caused by another pathology.

Our goal in this study is quantitatively evaluate the gray matter atrophy of patients with euthyroidism and hyperthyroidism of autoimmune etiology (Graves' disease) using Voxel-based Morphometry.

\section{Results and Discussion}

We acquired T1-weighted MRI images from 35 patients and 24 control subjects. Patients were separated into two groups according to the presence of active hyperthyroidism (15) or euthyroidism (20) and then these two groups were compared with the control group. We run VBM analyses using the standard protocol with CAT12 toolbox/SPM12 (http://www.neuro.unijena.de/cat/). We extracted volumetric grey matter maps through and performed group comparisons ANOVA $(p<0.001)$.

Grey matter atrophy was observed in the two groups (Figures 1/3, slightly more widespread in patients with hyperthyroidism) mainly in bilateral frontoparietal and in the temporal lobe, compared to controls. On the contrary, the excess of grey matter (Figures 2/4), was mainly identified in the cingulate gyrus in the hyperthyroidism and temporal lobe of euthyroid patients.

Despite the different clinical and laboratory manifestations of the two groups, volumes of grey matter marked with atrophy were similar and widespread. Besides, in the two groups, we identified grey matter excess, mainly in patients with hyperthyroidism.
Image 1. Grey matter atrophy in hyperthyroidism, mainly in the right temporal and occipital lobes.

Image 2. Grey matter excess in hyperthyroidism, mainly in the cingulate gyrus.

Image 3. Grey matter atrophy in euthyroidism, in the bilateral temporal lobe.

Image 4. Grey matter excess in euthyroidism, in the right temporal lobe.

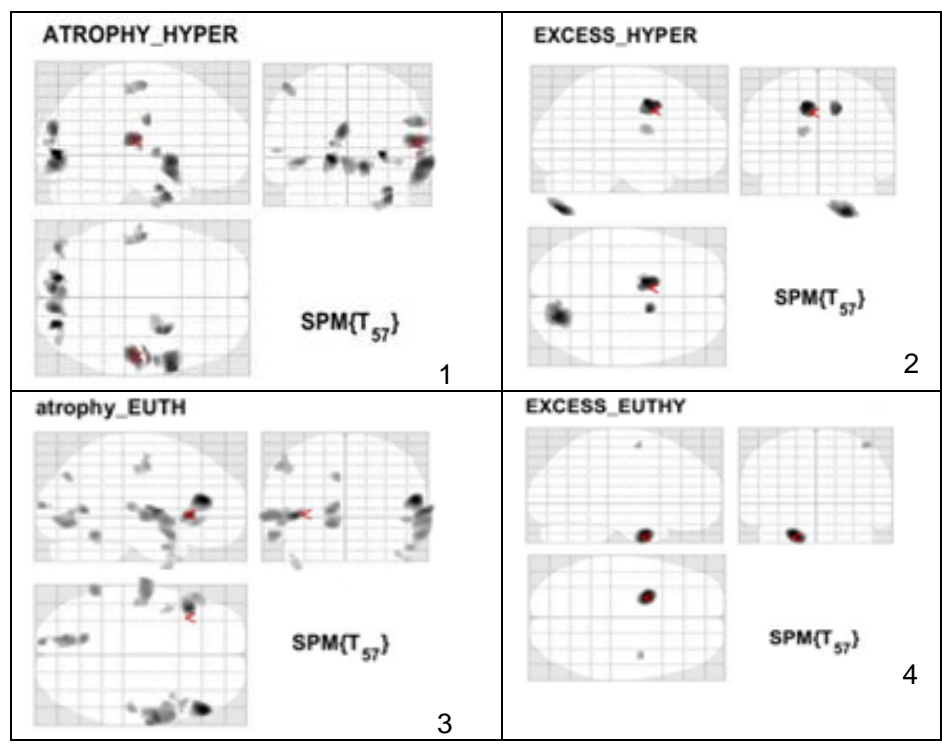

\section{Conclusions}

A comprehensive analysis of grey matter reinforces the negative impact of Grave's disease on brain atrophy, which persists even after resolution of thyrotoxicity.

\section{Acknowledgement}

I am thankful for Dr. Clarissa L. Yasuda, for the Laboratório de Neuroimagem (LNI), for the Hospital das Clínicas of UNICAMP and for the PIBIC/CNPq for the opportunity to present this study. Funded by CEPIDBRAINN (FAPESP) 2013/07559-3.

Khan FA, Al-Jameil N, Khan MF, Al-Rashid M, Tabassum H. Thyroid dysfunction: an autoimmune aspect. Int J Clin Exp Med. 2015;8(5):66776681

2 Blick C, Jialal I. Thyroid, Thyrotoxicosis. StatPearls [Internet]. Treasure Island (FL): StatPearls Publishing. 2018. 